\title{
Influence of multi-walled carbon nanotubes on the $\beta$ form crystallization of syndiotactic polystyrene at low temperature
}

\author{
A. Sorrentino*, L. Vertuccio, V. Vittoria \\ Chemical and Food Engineering Department - University of Salerno, via Ponte Don Melillo - 84084 Fisciano (SA), Italy
}

Received 28 December 2009; accepted in revised form 23 March 2010

\begin{abstract}
Syndiotactic polystyrene (sPS) samples were quenched at low temperature $\left(0^{\circ} \mathrm{C}\right)$ in the presence of Multi-Walled Carbon Nanotubes (MWCNT) at different concentrations. The influence of carbon nanotubes on the structure and physical properties of sPS composites was investigated by SEM, X-ray diffractograms, DSC (Differential Scanning Calorimetry), and Dynamic Mechanical Analysis (DMA). A good dispersion of the carbon nanotubes in the sPS matrix was found, at least for the low MWCNT concentration, whereas opposite effects were noticed on $T_{g}$ and elastic modulus, depending on concentration. The pristine sPS sample was almost amorphous at $0^{\circ} \mathrm{C}$, whereas in the filled samples the crystallinity increased on increasing the MWCNT concentration, indicating a strong nucleation effect of the nanotubes. Interestingly, in spite of the low temperature, the $\beta$ crystallinity was induced and this crystalline form increases on increasing the carbon nanotube concentration, being dominant (68\% of the total crystallinity) already at $3 \mathrm{wt} \%$ of MWCNT concentration.
\end{abstract}

Keywords: nanocomposites, polymer crystallization, multi-wall carbon nanotubes, syndiotactic polystyrene, morphology

\section{Introduction}

In semicrystalline polymer materials, the degree of crystallinity, the orientation, the polymorphism and the thermodynamic state of the amorphous phase are important factors in controlling the physical properties. Many efforts have been devoted in the past to settle a correlation picture between these parameters and the processing conditions [1]. This is particularly relevant for syndiotactic polystyrene (sPS) that presents a complex polymorphic pattern, depending on several factors, further complicated by the presence of structural disorder [2-5]. In particular, when crystallized from the molten state, sPS solidifies into two different crystal forms, termed $\alpha$ and $\beta$. They can appear pure or mixed in different proportions, depending on the experimental conditions $[1,6,7]$.
Controlling of processing conditions to obtain a pure $\alpha$ or $\beta$ form is of particular interest especially in consideration of their different physical properties $[2,6]$. For example, sPS samples in the crystalline $\alpha$ form, as well as amorphous samples, when exposed to suitable solvents transform into clathrate forms, characterized by chains in helical conformation. This transition never occurs in samples that present crystalline $\beta$ form [2]. For this reason, the sPS $\beta$ form could represent a polymeric material of industrial interest due to its high melting temperature, fast crystallization rate, good chemical and thermal resistance [2,5]. However, in spite of its thermodynamic higher stability with respect to the $\alpha$ form, it can be obtained only at very low under-cooling (high crystallization temperature), whereas at crystallization temperatures 
lower than $230^{\circ} \mathrm{C}$ the $\alpha$ form is always obtained [8].

Many attempts to obtain the $\beta$ form at lower temperatures failed so far. Polymorphic state has been only altered in the presence of miscible polymers, such as poly(2,6-dimethyl-p-phenylene oxide) [9] or atactic polystyrene, which reduced in sPS blends the $\alpha$ form crystallinity, increasing the $\beta$ form [10, $11]$.

Recent experimental investigations are demonstrating the potential of carbon nanotubes as small-volume fraction reinforcements in polymer matrix systems [12-17].

Carbon nanotubes are also known to impart profound changes to the crystallization behavior of semicrystalline polymers. They can affect the crystalline morphology [18] and modify the kinetics of the crystallization process as a result of their nucleating action [19-20]. Furthermore, carbon nanotubes may induce polymorphism in certain systems and promoting crystallization into specific crystal forms [21, 22].

In this paper we show the enhanced crystallization of sPS in the $\beta$ form, in the presence of multiwalled carbon nanotubes (MWCNT), even at very low crystallization temperatures. The possibility of inducing the $\beta$ form crystallization at low temperatures (close to the common processing conditions) is very important because in this way several polymer properties were improved, such as mechanical and thermal properties.

\section{Experimental}

\subsection{Materials}

Syndiotactic polystyrene (Questra QA101) was supplied by the Dow Chemical Co (USA). The molecular weight characteristics were: $M_{w}=$ $320000 \mathrm{~g} / \mathrm{mol}$ and $M_{w} / M_{n}=3.9$, whereas a concentration of syndiotactic triads was greater than $98 \%$ [6].

The carbon nanotubes were obtained from Nanocyl S. A. (Belgium) synthesized by catalytic carbon vapor deposition (ccvd) process. They are multiwalled carbon nanotubes characterized by an average diameter of about $10 \mathrm{~nm}$ and the length ranging from 0.1 to $10 \mu \mathrm{m}$. The nanotubes were purified to produce a carbon purity $>95 \%$ and a metal oxide impurity $<5 \%$ as it results by thermogravimetric analysis (TGA). The specific surface area deter- mined with the BET method is around 250$300 \mathrm{~m}_{2} / \mathrm{g}$ [17].

Neat sPS sample and the composite materials containing different concentration of multi-walled carbon nanotubes (1, 3, 6 and $9 \mathrm{wt} \%)$ were prepared using a laboratory-scale conical twin-screw extruder 'Micro-Coumpounder' by ThermoHaake (Germany). Polymers and carbon nanotubes were premixed manually and then loaded into the compounder. All batches, weighing $6 \mathrm{~g}$ each, were processed at $295^{\circ} \mathrm{C}$ with a screw speed of $100 \mathrm{rpm}$ for $15 \mathrm{~min}$. The samples were then extruded and cooled down to room temperature. The extruded samples were moulded in a hot press by Carver Inc. (USA) at $310^{\circ} \mathrm{C}$, forming $100 \mu \mathrm{m}$ thick films, which were rapidly quenched in a bath with water at $0^{\circ} \mathrm{C}$.

\subsection{Methods}

Differential Scanning Calorimetry (DSC) analysis was carried out on films samples with a mass ranging between 5 and $7 \mathrm{mg}$. The tests were carried out by means of a DSC 822 by Mettler Toledo (Germany) under nitrogen atmosphere. The samples were heated from 25 to $300^{\circ} \mathrm{C}$ at $10^{\circ} \mathrm{C} / \mathrm{min}$ and kept at $300^{\circ} \mathrm{C}$ for $5 \mathrm{~min}$ to eliminate the previous thermal history. After that, non isothermal meltcrystallization study was carried out by cooling the samples to $30^{\circ} \mathrm{C}$ at a rate of $10^{\circ} \mathrm{C} / \mathrm{min}$.

$\mathrm{X}$-ray diffraction (XRD) measurements were performed on the sample films by means of a $D 8$ Advance by Bruker (USA) X-ray diffractometer with $\mathrm{Ni}$-filtered $\mathrm{CuK}_{\alpha}$ radiation $(\lambda=1.5405 \AA)$. Data were collected from 2 to $40^{\circ}$ in $2 \theta$ in steps of $0.03^{\circ}$ at a time $/$ step of $0.02 \mathrm{sec}$.

Scanning electron microscopy (SEM) was conducted using a $J S M-5900 L V$ by JEOL (USA) scanning electron microscope. The films were etched before of the observation. The etching reagent was prepared by stirring $1.0 \mathrm{~g}$ potassium permanganate in a solution mixture of $95 \mathrm{ml}$ sulphuric acid (95-97\%) and $48 \mathrm{ml}$ orthophosphoric acid (85\%). The samples were immersed into the fresh etching reagent at room temperature and held under agitation for 48 hours. Subsequent washings were done using a cold mixture of 2 parts by volume of concentrated sulfuric acid and 7 parts of water. Furthermore the samples were washed successively with $30 \%$ aqueous hydrogen peroxide to remove 
any manganese dioxide. The samples were washed with distilled water and kept under vacuum for 2 days.

Thermo-mechanical properties of the different blends were determined with a dynamic thermomechanical analyzer, DMA 2980 by TA instrument (USA). Quenched film samples with dimensions $5 \times 10 \times 0.1 \mathrm{~mm}$ were tested by applying a variable tensile deformation. The displacement amplitude was set to $1 \%$, whereas the measurements were performed at the frequency of $1 \mathrm{~Hz}$. The range of temperature was from 0 to $150^{\circ} \mathrm{C}$ at a scanning rate of $3^{\circ} \mathrm{C} / \mathrm{min}$.

\section{Results and discussion}

\subsection{Samples morphology}

In Figure 1 the morphology of pristine sPS and the sample containing $1 \mathrm{wt} \%$ of MWCNT are compared. In the pristine sample the etching procedure erased the surface sheet, putting in evidence an almost structure-less morphology, typical of an amorphous sample.

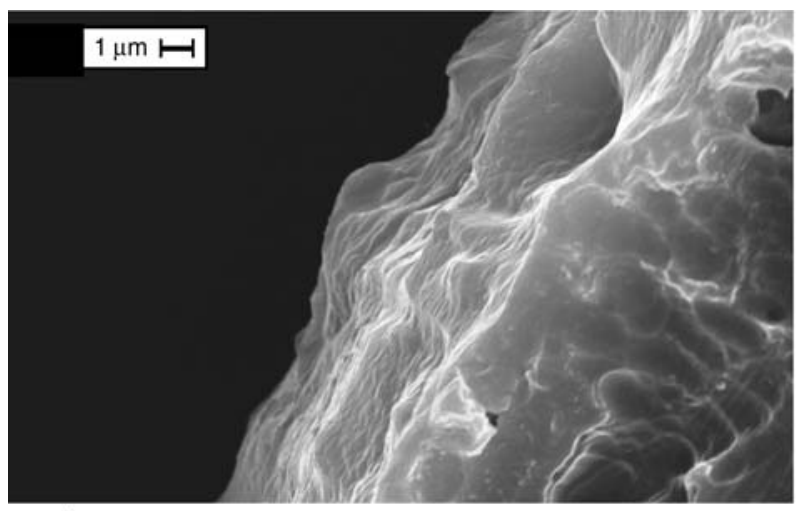

a)
The filled sample appears very similar, and no recognizable morphology is seen in the polymeric matrix; at variance a very homogeneous network of carbon nanotubes is clearly visible. They are nicely dispersed and do not show any bundle aggregate, going separately out of the polymeric matrix. This means that at the concentration of $1 \mathrm{wt} \%$ it is possible to disperse well the carbon nanotubes into the syndiotactic polystyrene matrix, producing a homogeneous sample. Further and deeper analyses, such as TEM, are needed to verify if the good dispersion is a general feature of the sPS-CNT composites, even for high concentrations.

\subsection{Differential Scanning Calorimetry (DSC)}

The DSC scans of sPS and sPS-MWCNT films quenched at $0^{\circ} \mathrm{C}$ are shown in Figure 2, during the first heating (Figure 2a) at $10^{\circ} \mathrm{C} / \mathrm{min}$ and successive cooling (Figure 2b) always at $10^{\circ} \mathrm{C} / \mathrm{min}$. In the heating of the neat sPS sample it is clearly observed a deflection from the baseline in the range 80$100^{\circ} \mathrm{C}$, due to the amorphous glass transition, an

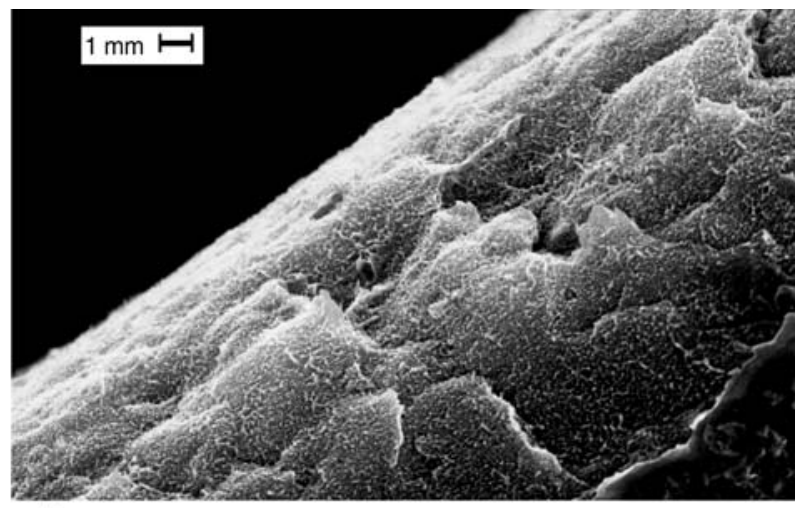

b)

Figure 1. Scanning electron microscopy of selected samples. a) neat sPS; b) sPS containing 1 wt $\%$ of MWCNT
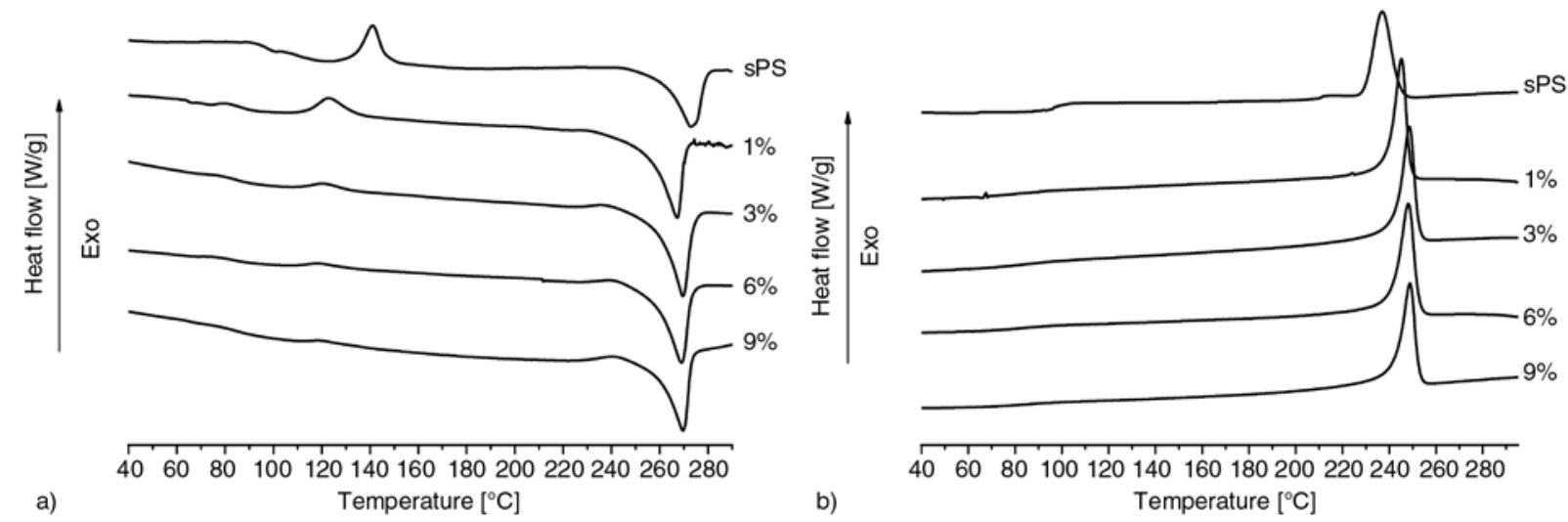

Figure 2. DSC curves of the reported samples: a) heating scan at $10^{\circ} \mathrm{C} / \mathrm{min}$ of the quenched samples; b) cooling scan at $10^{\circ} \mathrm{C} / \mathrm{min}$ of the same samples after the annealing step at $300^{\circ} \mathrm{C}$ 
exothermic peak due to the cold crystallization and the melting peak. The jump at the glass transition temperature $\left(T_{g}\right)$ is quite evident in the pristine sPS sample, whereas it appears at lower temperatures and is less evident in the composites. The subsequent exothermic peak due to the cold crystallization indicates that the pure sPS films quenched at $0^{\circ} \mathrm{C}$ is prevalently amorphous and crystallizes during the heating scan. At variance this peak is reduced in the sample containing MWCNT up to fully disappear in the samples with 6 and $9 \mathrm{wt} \%$ of MWCNT.

It is interesting to note that the cold crystallization temperature $\left(T_{c \text { (heating) }}\right)$ (the temperature of the exothermic peak) decreases by almost $20^{\circ} \mathrm{C}$ going from the pure to the composite samples, indicating a nucleation effect due to the carbon nanotubes $[19,20]$.

This effect is also detectable during the cooling scan from the melt state, in which the crystallization temperature $\left(T_{c(\text { cooling })}\right)$ of the composite samples is always higher than that of the neat sPS.

The melting temperatures $\left(T_{m}\right)$ are slightly lower in the composite samples. This is due to the fact that the crystals formed during the DSC heating scan have a melting temperature higher than that formed during the rapid quenching at $0^{\circ} \mathrm{C}$.

All the thermal parameters are reported in Table 1, in which also the crystallinity $\left(X_{c}\right)$, evaluated using the following Equation (1) is reported:

$$
X_{c}=\frac{\Delta H_{m}-\Delta H_{c}}{\Delta H_{\infty}} \cdot 100
$$

where $\Delta H_{c}$ and $\Delta H_{m}$ are the enthalpy of cold crystallization and fusion recorded by DSC, and $\Delta H_{\infty}$ is the heat of fusion for $100 \%$ crystalline sPS (taken to be $53.2 \mathrm{~J} / \mathrm{g}$ [23]).

The enthalpies, and consequently the crystallization degree, reported in Table 1, are normalized to the polymer mass.

It is important to note that this method tends to overestimate the value of crystallinity in presence of mesophase and/or melting - re-crystallization processes during the heating scan at temperatures close to the melting point. In particular, the formations of an instable mesophase during the crystallization of sPS at lower temperature, was clearly observed in the literature $[2,8]$. Even if the conformational order of the chain sequences is not sufficient to give rise to discrete $\mathrm{X}$-ray reflections, the presence of mesomorphic phase can be characterized by infrared spectroscopy as well as permeability tests [8].

It is also evident that increasing the MWCNT concentration the crystallinity increases, being almost double with respect to the pure sPS for the samples with 3, 6 and 9 wt\% nanotubes. The shift of the glass transition temperature at lower temperatures is also worth noting in all the composite samples. Probably, as observed in other polymer nanocomposites systems, the presence of CNT in the matrix induces an increase of free volume, and consequently an increase in the molecular mobility at lower temperature $[24,25]$.

\subsection{X-ray analysis}

In Figure 3 the X-ray diffractograms of the pure sPS and all the sPS-MWCNT composites are shown. The pure sPS sample is almost completely

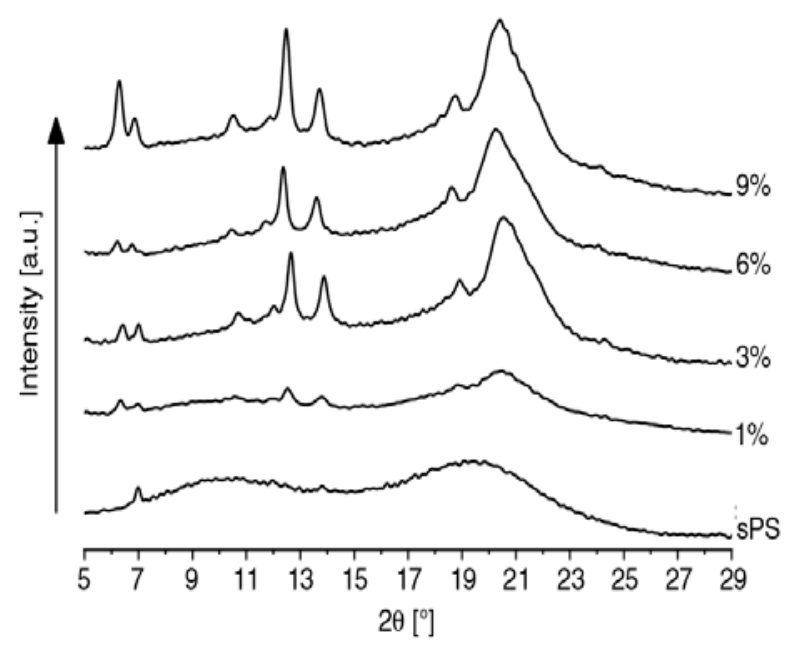

Figure 3. X-ray powder spectra of the reported samples

Table 1. DSC results for the reported samples

\begin{tabular}{|c|c|c|c|c|c|c|c|c|}
\hline \% MWCNT & $\begin{array}{c}\mathbf{T}_{\mathbf{g}} \\
{\left[{ }^{\circ} \mathbf{C}\right]}\end{array}$ & $\begin{array}{c}\mathbf{T}_{\mathbf{c}(\text { heating })} \\
{\left[{ }^{\circ} \mathbf{C}\right]}\end{array}$ & $\begin{array}{c}\Delta \mathbf{H}_{\mathrm{c}(\text { heating })} \\
{[\mathrm{J} / \mathrm{g}]}\end{array}$ & $\begin{array}{c}\mathbf{T}_{\mathbf{m}} \\
{\left[{ }^{\circ} \mathbf{C}\right]}\end{array}$ & $\begin{array}{l}\Delta \mathbf{H}_{\mathrm{m}} \\
{[\mathrm{J} / \mathrm{g}]}\end{array}$ & $\begin{array}{c}\mathbf{X}_{\mathbf{c}} \\
{[\%]}\end{array}$ & $\begin{array}{c}\mathbf{T}_{\mathbf{c}(\text { cooling) }} \\
{\left[{ }^{\circ} \mathbf{C}\right]}\end{array}$ & $\begin{array}{c}\Delta \mathbf{H}_{\mathbf{c}(\text { cooling) }} \\
{[\mathbf{J} / \mathbf{g}]}\end{array}$ \\
\hline sPS & 97.5 & 141 & 11.26 & 273 & 24.29 & 24 & 237 & 24.30 \\
\hline 1 & 81.0 & 123 & 7.42 & 267 & 28.91 & 40 & 245 & 26.78 \\
\hline 3 & 84.0 & 120 & 2.51 & 270 & 30.46 & 52 & 248 & 29.44 \\
\hline 6 & 85.0 & 119 & 1.44 & 269 & 29.04 & 52 & 248 & 28.52 \\
\hline 9 & 83.0 & 120 & 1.29 & 269 & 30.89 & 56 & 249 & 27.32 \\
\hline
\end{tabular}


amorphous: indeed only a small peak at $6.8^{\circ}$ of $2 \theta$, characteristic of the $\alpha$ form $[2,3]$ is apparent in the diffractogram. This is an expected result, always found for quenching at low temperatures.

At variance, all the composites appear crystalline, with this parameter increasing on increasing the carbon nanotube concentration, confirming the DSC results. Moreover the crystalline diffractograms surprisingly show the presence of peaks characteristic of the $\beta$ form, never observed at crystallization temperature as low as $0^{\circ} \mathrm{C}$. Indeed the peaks at 6.2 and $12.3^{\circ}$ of $2 \theta$ typical of the $\beta$ form $[2,3]$ are evident in the sample containing $1 \mathrm{wt} \%$ of MWCNT, and their intensity increases on increasing the nanotube concentration. Also the peaks characteristic of the $\alpha$ form are present in the diffractograms, in particular the peaks at 6.8 and $11.8^{\circ}$ of $2 \theta[2,3]$. However, at variance with the $\beta$ form peaks, the $\alpha$ form peaks decrease on increasing the nanotube concentration, indicating that the nanotubes strongly favor the $\beta$ crystallization. The total crystallinity was derived from the diffractograms by comparing the crystalline peak area with the total area of the diffractograms. The obtained values are reported in Table 2. The X-ray analysis, which represents the crystallinity of the quenched samples (not invalidated by further crystallization as in DSC), shows a dramatic increase of crystallinity from the pure sPS sample (3\%) and the sample with $3 \mathrm{wt} \%$ of MWCNT (36\%), confirming the qualitative behavior deduced in the DSC analysis.

To investigate the variation of the $\beta$ form crystallinity on increasing the nanotube concentration, the formula of Guerra et al. [8] was used for calculating the $\beta$ fraction.

It consists of determining the areas $(I)$ of the two peaks located at $2 \theta=11.6^{\circ}$ ( $\alpha$ form) and $2 \theta=12.2$ ( $\beta$ form), and deriving the percentage of $\beta$ form by the Equation (2) where 1.8 is ratio between the intensities of peaks at 11.6 and $12.2^{\circ}$ of $2 \theta$, for sample of equal thickness and crystallinity in the pure $\alpha$ and $\beta$ form, respectively:

Table 2. XRD results for the reported samples

\begin{tabular}{|c|c|c|}
\hline \% MWCNT & $\mathbf{X}_{\mathbf{c}}[\boldsymbol{\%}]$ & $\mathbf{P}_{\boldsymbol{\beta}}[\boldsymbol{\%}]$ \\
\hline sPS & 3 & 0 \\
\hline 1 & 18 & 33 \\
\hline 3 & 36 & 68 \\
\hline 6 & 34 & 77 \\
\hline 9 & 39 & 83 \\
\hline
\end{tabular}

$$
P_{\beta}=\left[1-\frac{\frac{1.8 \cdot I_{\left(11.6^{\circ}\right)}}{I_{\left(12.2^{\circ}\right)}}}{1+\frac{1.8 \cdot I_{\left(11.6^{\circ}\right)}}{I_{\left(12.2^{\circ}\right)}}}\right] \cdot 100
$$

In Table 2 the also the relative $P_{\beta}$ crystallinity are reported. A strong increase of both the total and the $\beta$ crystallinity is clearly observed on increasing the carbon nanotube concentration, with the $\beta$ form being dominant ( $68 \%$ of the total crystallinity) already at $3 \mathrm{wt} \%$ of MWCNT concentration.

\subsection{Dynamic mechanical properties}

Figure 4 shows the tensile storage modulus $\left(E^{\prime}\right)$ and the glass transition temperature $\left(T_{g}\right)$, resulting from the DMA characterization of the samples.

MWCNT addition increases the storage modulus of the composites for all the concentrations. The glass transition temperature (identified as the temperature where the tan $\delta$ presents a peak) after a sudden decrease, increases on increasing the MWCNT concentration. Even if this trend was confirmed by the experimental results found in the DSC analysis (Figure 2a), it is difficult to explain. It probably corresponds to an overlapping of different effects related to the free volume, the crystallinity of sPS matrix, the relaxation of the rigid amorphous phase immobilized by carbon nanotubes and inter-phase interactions [26]. At low CNT concentration the increase of free volume, determining an increase of chain mobility prevails, determining a decrease of the $T_{g}$, whereas at high CNT concentration the

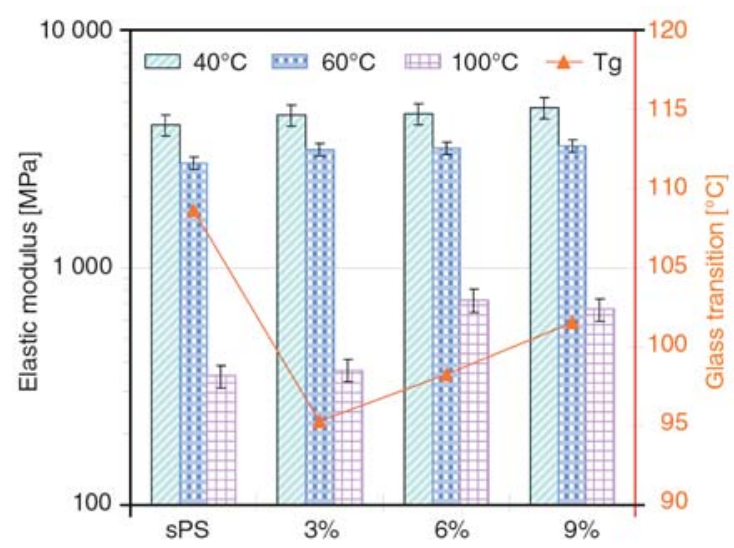

Figure 4. Storage modulus at 40,60 and $100^{\circ} \mathrm{C}$ and glass transition temperature as a function of nanotubes concentration as calculated by the DMA tensile tests 
increasing amount of filler increases the restrictions imposed to the motion of the polymer chains, which can explain the increase after the initial drop. The decrease in the chain mobility and principally the consistent increase of crystallinity can explain also the enhancement in the elastic modulus. A similar behaviour was described in [27] for another semicrystalline polymer-nanocomposite system (PA6-MWCNT composites).

\section{Conclusions}

The influence of carbon nanotubes on the structure and physical properties of sPS composites has been investigated by different methods, giving the following results:

- The chosen processing conditions allows, at least for low concentrations, a good dispersion of the carbon nanotubes in the sPS matrix.

- As evidenced by DMA and DSC analysis, the glass transition decreases in the filled samples, indicating that the effect of carbon nanotubes on the amorphous phase of sPS produce different and opposing effects.

- The pristine sPS sample is almost amorphous at $0^{\circ} \mathrm{C}$, whereas in the filled samples the crystallinity increases on increasing the MWCNT concentration, indicating a strong nucleation effect of the nanotubes.

- The $\beta$ crystallinity increases on increasing the carbon nanotube concentration, being dominant (68\% of the total crystallinity) already at $3 \mathrm{wt} \%$ of MWCNT concentration.

- MWCNT addition increases the storage modulus of the composites for all the concentrations.

\section{References}

[1] Sorrentino A., De Santis F., Titomanlio G.: Polymer crystallization under high cooling rate and pressure: A step towards polymer processing conditions. in 'Lecture notes in physics: Progress in understanding of polymer crystallization' (eds.: Reiter G., Strobl G.) Springer, Berlin, Vol 714, 329-344 (2007).

[2] Sorrentino A., Vittoria V.: Structure, morphology, and crystallization behavior of syndiotactic polystyrene. in 'Syndiotactic polystyrene: Synthesis, characterization, processing, and applications' (ed.: Schellenberg J.) Wiley, Chichester, 157-194 (2009).
[3] De Rosa C., de Ballesteros O. R., Di Gennaro, M., Auriemma, F.: Crystallization from the melt of $\alpha$ and $\beta$ forms of syndiotactic polystyrene. Polymer, 44, 1861-1870 (2003). DOI: $\underline{10.1016 / \mathrm{S} 0032-3861(02) 00885-6}$

[4] De Rosa C.: Crystal structure of the trigonal modification ( $\alpha$ form) of syndiotactic polystyrene. Macromolecules, 29, 8460-8465 (1996).

DOI: $10.1021 / \mathrm{ma} 960905 \mathrm{q}$

[5] Greis O., Asano T., Xu Y., Petermann J.: Morphology and structure of syndiotactic polystyrene. Polymer, 30, 590-594 (1989). DOI: 10.1016/0032-3861(89)90140-7

[6] Sorrentino A., Pantani R., Titomanlio G.: Kinetics of melting and characterization of the thermodynamic and kinetic properties of syndiotactic polystyrene. Journal of Polymer Science Part B: Polymer Physics, 45, 196-207 (2007). DOI: $10.1002 /$ polb.21039

[7] Sorrentino A., Pantani R., Titomanlio G.: Melting and zero growth rate temperatures of syndiotactic polystryrene. Colloid and Polymer Science, 286, 983 991 (2008). DOI: $\underline{10.1007 / \mathrm{s} 00396-008-1858-2}$

[8] Guerra G., Vitagliano V., De Rosa C., Petraccone V., Corradini P.: Polymorphism in melt crystallized syndiotactic polystyrene samples. Macromolecules, 23, 1539-1544 (1990).

DOI: $10.1021 / \mathrm{ma} 00207 \mathrm{a} 050$

[9] Dikshit A. K., Kaito A.: Effects of the oriented mesophase on the cold crystallization of syndiotactic polystyrene and its blend with poly(2,6-dimethyl-1,4phenylene oxide). Journal of Polymer Science Part B: Polymer Physics, 41, 1665-1675 (2003).

DOI: $\underline{10.1002 / \text { polb. } 10520}$

[10] Woo E. M., Sun Y. S., Yang C-P.: Polymorphism, thermal behavior, and crystal stability in syndiotactic polystyrene vs. its miscible blends. Progress in Polymer Science, 26, 945-983 (2001). DOI: 10.1016/S0079-6700(01)00010-7

[11] Guerra G., De Rosa C., Vitagliano V. M., Petraccone V., Corradini P.: Effects of blending on the polymorphic behavior of melt-crystallized syndiotactic polystyrene. Journal of Polymer Science Part B: Polymer Physics, 29, 265-271 (1991). DOI: 10.1002/polb.1991.090290301

[12] Shaffer M. S. P., Windle A. H.: Fabrication and characterization of carbon nanotube/poly(vinyl alcohol) composites. Advanced Material, 11, 937-941 (1999). DOI: $10.1002 /($ SICI) 1521-4095(199908)11:11<937:: AID-ADMA937>3.0.CO;2-9

[13] Quian D., Dickey E. C., Andrews R., Rantell T.: Load transfer and deformation mechanisms in carbon nanotube-polystyrene composites. Applied Physic Letters, 76, 2868-2870 (2000).

DOI: $\underline{10.1063 / 1.126500}$ 
[14] Kashiwagi T., Grulke E., Hilding J., Harris R., Awad W., Douglas J.: Thermal degradation and flammability properties of poly(propylene)/carbon nanotube composites. Macromolecular Rapid Communication, 23, 761-765 (2002).

DOI: 10.1002/1521-3927(20020901)23:13<761::AID-

\section{MARC761>3.0.CO;2-K}

[15] Andrews R., Weisenberger M. C.: Carbon nanotube polymer composites. Current Opinion in Solid State and Materials Science, 8, 31-37 (2004).

DOI: $\underline{10.1016 / \mathrm{j} . c o s s m s .2003 .10 .006}$

[16] Ajayan P. M., Zhou O. Z.: Applications of carbon nanotubes. in 'Carbon nanotubes: Synthesis, structure, properties, and applications' (eds.: Dresselhaus M. S., Dresselhaus G., Avouris P.) Springer, Berlin, 386-421 (2000).

[17] Guadagno L., Vertuccio L., Sorrentino A., Raimondo M., Naddeo C., Vittoria V., Iannuzzo G., Calvi E., Russo S.: Mechanical and barrier properties of epoxy resin filled with multi-walled carbon nanotubes. Carbon, 47, 2419-2430 (2009).

DOI: $10.1016 /$ j.carbon.2009.04.035

[18] Jeon K., Lumata L., Tokumoto T., Steven E., Brooks J., Alamo R. G.: Low electrical conductivity threshold and crystalline morphology of single-walled carbon nanotubes - High density polyethylene nanocomposites characterized by SEM, Raman spectroscopy and AFM. Polymer, 48, 4751-4764 (2007).

DOI: $10.1016 /$ j.polymer.2007.05.078

[19] Sandler J. K. W., Pegel S., Cadek M., Gojny F. H., van Es M., Lohmar J., Blau W. J., Schulte K., Windle A. H., Shaffer M. S. P.: A comparative study of melt spun polyamide-12 fibres reinforced with carbon nanotubes and nanofibres. Polymer, 45, 2001-2015 (2004).

DOI: $10.1016 /$ j.polymer.2004.01.023

[20] Probst O., Moore E. M., Resasco D. E., Grady B. P.: Nucleation of polyvinyl alcohol crystallization by single-walled carbon nanotubes. Polymer, 45, 44374443 (2004).

DOI: $10.1016 /$ j.polymer.2004.04.031
[21] Sarno M., Gorrasi G., Sannino D., Sorrentino A., Ciambelli P., Vittoria V.: Polymorphism and thermal behaviour of syndiotactic poly(propylene)/carbon nanotube composites. Macromolecular Rapid Communications, 25, 1963-1967 (2004).

DOI: $10.1002 / \mathrm{marc} .200400344$

[22] Li J. A., Fang Z. P., Tong L. F., Gu A. J., Liu F.: Polymorphism of nylon-6 in multiwalled carbon nanotubes/nylon-6 composites. Journal of Polymer Science Part B: Polymer Physics, 44, 1499-1512 (2006).

DOI: $10.1002 /$ polb.20808

[23] Pasztor A. J., Landes B. G., Karjala P. J.: Thermal properties of syndiotactic polystyrene. Thermochimica Acta, 177, 187-195 (1991).

DOI: $10.1016 / 0040-6031(91) 80095-Z$

[24] Drozdov A. D., Christiansen J. C., Gupta R. K., Shah A. P.: Model for anomalous moisture diffusion through a polymer-clay nanocomposite. Journal of Polymer Science Part B: Polymer Physics, 41, 476492 (2003).

DOI: $10.1002 /$ polb. 10393

[25] Sorrentino A., Gorrasi G., Tortora M., Vittoria V.: Barrier properties of polymer/clay nanocomposites. in 'Polymer nanocomposites' (eds.: Mai Y-W., Yu Z-Z.) Woodhead, Cambridge (273-292) (2006).

[26] Pantani R., Sorrentino A.: Pressure effect on viscosity for atactic and syndiotactic polystyrene. Polymer Bulletin, 54, 365-376 (2005). DOI: $10.1007 / \mathrm{s} 00289-005-0397-\mathrm{y}$

[27] Logakis E., Pandis C., Peoglos V., Pissis P., Stergiou C., Pionteck J., Pötschke P., Micušík M., Omastová M.: Structure-property relationships in polyamide 6/multi-walled carbon nanotubes nanocomposites. Journal of Polymer Science Part B: Polymer Physics, 47, 764-774 (2009).

DOI: $10.1002 /$ polb. 21681 